\title{
An Atomic-Scale Picture of the Composition, Decay and Oxidation of Two-Dimensional Radioactive Films
}

\author{
Alex Pronschinske ${ }^{1}$, Philipp Pedevilla ${ }^{2}$, Benjamin Coughlin ${ }^{1}$, Colin J. Murphy ${ }^{1}$, \\ Felicia R. Lucci ${ }^{1}$, Matthew A. Payne ${ }^{3}$, Andrew J. Gellman ${ }^{3}$, \\ Angelos Michaelides ${ }^{2 *}$, and E. Charles H. Sykes ${ }^{1 *}$ \\ ${ }^{1}$ Department of Chemistry, Tufts University, 62 Talbot Avenue, Medford, MA 02155, USA. \\ ${ }^{2}$ Thomas Young Centre, London Centre for Nanotechnology and Department of Chemistry, University \\ College London, London WC1E 6BT, UK. \\ ${ }^{3}$ Department of Chemical Engineering, Carnegie Mellon University, Pittsburgh, PA 15213, USA
}

* To whom correspondence should be addressed: angelos.michaelides@ucl.ac.uk,charles.sykes@tufts.edu

RECEIVED DATE:

KEYWORDS: Radioactive, 2D material, self-sustaining electron source, iodine-125, electron emission, STM 


\section{ABSTRACT:}

2D radioactive ${ }^{125} \mathrm{I}$ monolayers are a recent development that combine the fields of radiochemistry and nanoscience. These Au supported monolayers show great promise for understanding the local interaction of radiation with 2D molecular layers, offer different directions for surface patterning, and enhance the emission of chemically and biologically relevant low energy electrons. However, the elemental composition of these monolayers is in constant flux due to the nuclear transmutation of ${ }^{125} \mathrm{I}$ to ${ }^{125} \mathrm{Te}$ and their precise composition and stability under ambient conditions has yet to be elucidated. Unlike I, which is stable and unreactive when bound to $\mathrm{Au}$, the newly formed Te atoms would be expected to be more reactive. We have used electron emission and X-ray photoelectron spectroscopy (XPS) to quantify the emitted electron energies, track the film composition in vacuum and the effect of exposure to ambient conditions. Our results reveal that the Auger electrons emitted during the ultra-fast radioactive decay process have a kinetic energy corresponding to neutral Te. By combining XPS and scanning tunneling microscopy experiments with density functional theory we are able to identify the reaction of newly formed $\mathrm{Te}$ to $\mathrm{TeO}_{2}$ and its subsequent dimerization. The fact that the $\mathrm{Te}_{2} \mathrm{O}_{4}$ units stay intact during major lateral rearrangement of the monolayer illustrates their stability. These results provide an atomic-scale picture of the composition and mobility of surface species in a radioactive monolayer as well as an understanding of the stability of the films under ambient conditions, which is a critical aspect in their future applications. 
The interaction of high energy radiation ( $\gamma$-rays, $X$-rays, $\alpha$ and $\beta$ particles) with matter produces not only primary impact damage, but also large numbers of non-thermal secondary electrons $(<10 \mathrm{eV})$ along the radiation track. ${ }^{1-3}$ Although these electrons are below the ionization energies of biologically relevant molecules, they initiate radiationinduced chemical reactions, making them arguably the most important species in radiation chemistry. ${ }^{2,45}$ The bond breaking mechanism at energies less than $10 \mathrm{eV}$ is primarily from dissociative electron attachment, during which a short-lived negative ion of the molecule is formed and then dissociates into a radical fragment and an anionic fragment. ${ }^{6,7}$ At higher energies, electron impact excitation $(>6 \mathrm{eV}$ ) and ionization (> 10 $\mathrm{eV}$ ) events begin to occur. Even if the electron does not react directly with e.g. a DNA molecule, indirect effects such as the formation of solvated electrons can lead to genome damage. ${ }^{8-11}$ As a result of their high reactivity and short path length, any source of low energy electrons must be within $\sim 10 \mathrm{~nm}$ of a target to induce reaction chemistry. ${ }^{12}$ As a method to escape the need for an external radiation source to power the production of low energy electrons, Pronschinske et al. reported on single-atom thick, ordered radioactive films of ${ }^{125} \mathrm{I}$ on gold that can be constructed on the same nanometer scale as a low-energy electron's range. These films provide a self-sustaining source of copious amounts of low energy electrons in the $0-10 \mathrm{eV}$ range. ${ }^{1}$ Electrons in this low energy range have been shown to cause both DNA single and double strand breaks in electron stimulated desorption experiments and are also implicated in the increased anti-tumor efficacy of gold nanoparticles irradiated with X-rays. ${ }^{13-16}$ As a first step towards application of this new approach for local low energy electron delivery, e.g. conjugated to gold nanoparticles, ${ }^{17-23}$ characteristics such as the radioactive film stability and reactivity in an ambient environment must be understood.

In terms of applications as a self-sustaining nano-emitter, given the massive amount of energy released during the nuclear decay of radioactive ${ }^{125} \mathrm{I}(185 \mathrm{keV})$ one must consider the stability of the material before, during, and after nuclear decay as well as the effect of ambient conditions. The ${ }^{125} \mathrm{I} / \mathrm{Au}$ combination is well suited for application because of the strong I-Au bond ${ }^{24,25}$ and negligible neutrino-induced nuclear recoil of ${ }^{125} \mathrm{I}(0.1 \mathrm{eV})$. This recoil energy is very low compared to other isotopes that decay by electron capture (EC), 
or through emission of an $\alpha$ or $\beta$ particle. ${ }^{26-30}$ The decay of ${ }^{125}$ I via EC results in a nuclear proton and a core-level electron being converted to a neutron with the release of an electron neutrino, and hence elemental transmutation of ${ }^{125} \mathrm{I}$ into ${ }^{125} \mathrm{Te}$. While there have been some studies of Te films on $\mathrm{Au}(111)$, they have mostly focused on depositing the element with electrochemical control. ${ }^{31,32}$ Another potential chemical instability resulting from nuclear decay is Coulomb explosion that results from slow neutralization of the radionuclide following ultra-fast emission of copious amounts of its valence electrons, ${ }^{33-}$ ${ }^{36}$ which for ${ }^{125}$ I can be upwards of 20 electrons as predicted by Monte Carlo studies. ${ }^{37}$ When such highly charged radionuclides have insufficient electronic coupling to their environment, for example ${ }^{55} \mathrm{Fe}$-containing alkanethiol SAMs, their rate of neutralization falls below the timescale of atomic motion. This leads to neighboring atoms becoming positively charged and causing local damage through electrostatic repulsion and ejection of ions and charged molecular fragments. ${ }^{33}$

In this study we use X-ray photoelectron spectroscopy (XPS) to probe the surface composition of radioactive ${ }^{125} \mathrm{I} / \mathrm{Au}$ films over time and after exposure to air. This technique allows us to quantify the elemental species and their oxidation states with high surface sensitivity. ${ }^{38}$ Density functional theory (DFT) calculations were employed to quantify the binding strength of both the radionuclide ${ }^{125} \mathrm{I}$ and daughter ${ }^{125} \mathrm{Te}$ to the $\mathrm{Au}$ substrate. Furthermore, we used DFT calculations to examine the reactivity of the newly formed Te atoms with a range of common molecules present under ambient conditions. This work revealed that the most exothermic reaction involved oxidation of $\mathrm{Te}$ to $\mathrm{TeO}_{2}$. This oxidized Te (in a Te ${ }^{4+}$ oxidation state) was observed by XPS after film exposure to ambient conditions and scanning tunneling microscopy (STM) revealed the presence of isolated dimers in the film which DFT predicted are thermodynamically preferred $\mathrm{Te}_{2} \mathrm{O}_{4}$ species formed by two interacting $\mathrm{TeO}_{2}$ units. Overall, these results look promising in terms of future applications of ${ }^{125} \mathrm{I} / \mathrm{Au}$ devices as both the radionuclide and daughter product remain strongly bound to the Au surface during both nuclear decay and exposure to ambient conditions.

\section{RESULTS AND DISCUSSION}


${ }^{125} \mathrm{I} / \mathrm{Au}(111)$ samples were made using the established ambient drop-casting method at PerkinElmer (Billerica, MA); a photograph of the drop-casting procedure is shown in Figure 1a. ${ }^{1}$ The samples were then transferred to Tufts and Carnegie Mellon Universities under ambient conditions over a period of 1-2 days. Figure 1b shows a high-resolution STM image of a sample in which the I atoms of the monolayer film are clearly resolved. Single atom depression are randomly distributed in the monolayer structure that correspond to Te atoms formed from the nuclear transmutation of ${ }^{125} \mathrm{I}^{1}$ Given the copious amounts of electrons that are emitted by these samples we will first discuss our electron emission studies that report on the charge state of the decaying atoms before moving on to measurements of the composition and oxidation by XPS and DFT modeling of various reaction pathways.

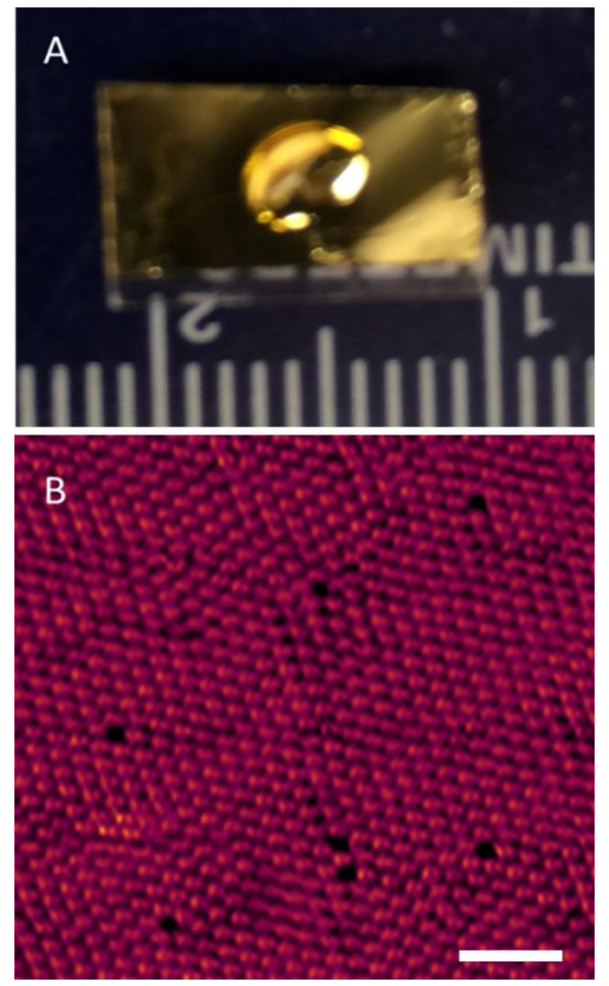

Figure 1 (A) Photograph of the deposition of an aqueous solution of $\mathrm{Na}^{125} \mathrm{I}(161 \mu \mathrm{M})$ on Au(111) using an ambient dropcasting method (B) The resulting ${ }^{125} \mathrm{I}$ monolayer as imaged using scanning tunneling microscopy $(\mathrm{V}=-0.4 \mathrm{~V}, \mathrm{I}=0.1 \mathrm{nA}$, scale bar $=2 \mathrm{~nm})$. The radioactive decay of ${ }^{125} \mathrm{I}$ (half-life $=60$ days) is a multi-step process consisting of (in order) EC, an electron emission cascade, relaxation of the excited ${ }^{125} \mathrm{Te}$ nucleus, and a second electron emission cascade. These cascades are driven by Auger relaxations, two- 
electron processes in which an ejected electron leaves behind a hole which is filled by a higher lying electron and the additional energy is released in the form of a second electron. This second electron is called the Auger electron and has a kinetic energy dependent only on the charge state of the atom and not the initial excitation energy. The MNN Auger transition of the element Te is lower in energy than most of the emitted electrons and typically occurs near the end of the electron emission cascade. By placing the ${ }^{125} \mathrm{I} / \mathrm{Au}$ samples in ultra-high vacuum we are able to use a concentric hemispherical electron energy analyzer to measure the energy of these Auger electrons and gain a window into the charge state of the newly formed Te atom during the ultra-fast electron emission cascades. Electron emission from radioactive decay of ${ }^{125} \mathrm{I}$ occurs in two stages. ${ }^{46}$ Briefly, in the first step called electron capture a core electron tunnels into the nucleus and combines with a proton. This leaves a core hole which releases its energy in a 0.1 -10 fs timescale via X-rays and Auger electrons. The Te nucleus is left in an excited state with a half-life of $\sim 2$ ns which, 7\% of the time, decays via gamma ray emission. In the other $93 \%$ of cases the excess nuclear energy is released via ejection of an inner shell electron, leaving another core hole which decays via a similar cascade of events to the first step. Therefore, as mentioned in the introduction, charging of the newly formed Te atom depends on the relative rates of electron emission and neutralization from surrounding Au atoms. Given that Auger emission is dependent only on the charge state of the atom and not the source of the initial excitation, the kinetic energy of these transitions provide direct insight into how fast the newly formed Te atom can neutralize during these ultra-fast cascades.

In Figure 2 we observe the Te MNN Auger electron emission peaks at kinetic energies of 483 and $493 \mathrm{eV}$, which is at the same energy as a neutral Te atom. ${ }^{47}$ Based on literature photoemission data, one can expect that the Te MNN Auger peak will shift by around $-1 \mathrm{eV}$ (in kinetic energy) for each additional +1 charge on the Te atom. ${ }^{47}$ Monte Carlo studies of the decay of ${ }^{125} \mathrm{I}$ atoms have shown that, on average, 13 electrons are ejected per decay event ${ }^{33}$. Therefore, if the ${ }^{125}$ Te were charging up to +13 after being formed from ${ }^{125} \mathrm{I}$, the Te MNN Auger peak would appear drastically shifted to around $470 \mathrm{eV}$. However, it is clear from the data in Figure 2 that despite the ultra-fast electron emission 
cascades following a ${ }^{125}$ I EC event, the daughter Te atom does not charge up. In addition to the two predominant $\mathrm{Te}^{0}$ MNN Auger electron emission peaks we observe a smaller pair of peaks at 503 and $514 \mathrm{eV}$ corresponding to I MNN Auger electron emission peaks. This would infer that some of the surrounding ${ }^{125} \mathrm{I}$ atoms that have not yet decayed are excited by radiation in the form of either photons or electrons emitted during the radioactive decay.

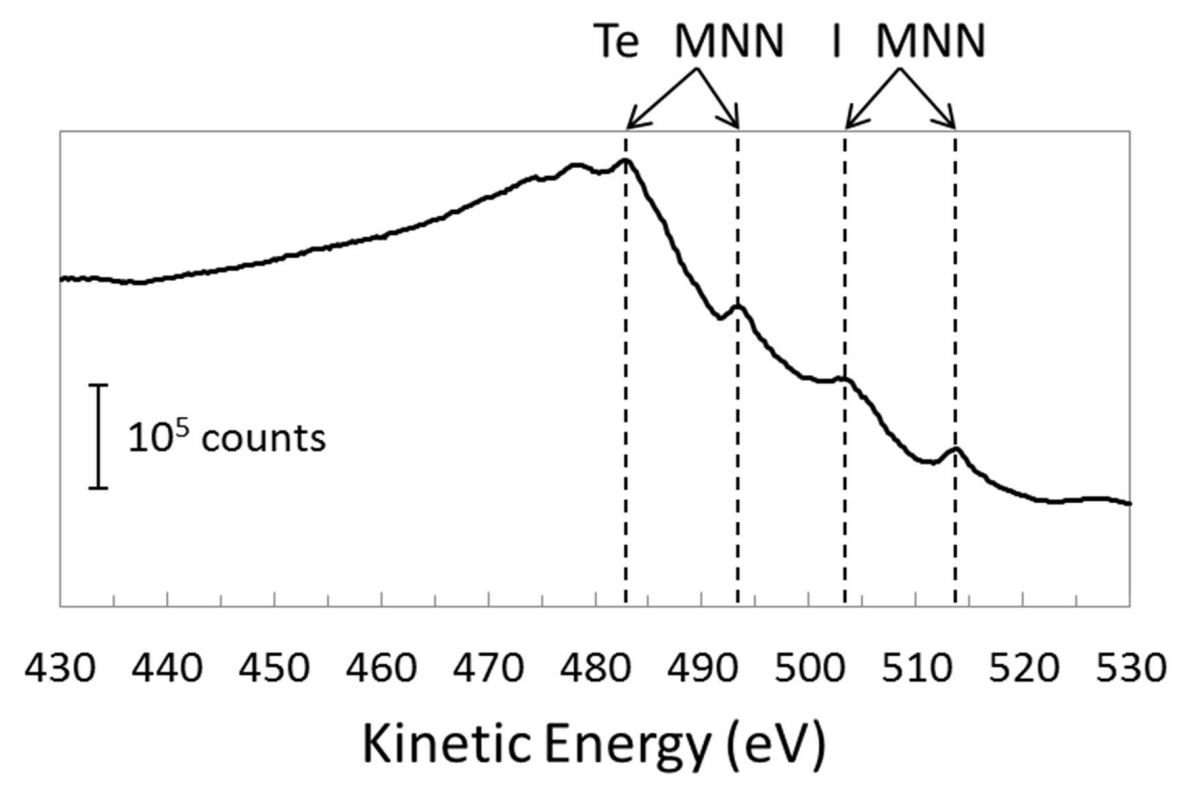

Figure 2 Kinetic energy spectrum of electron emission during radioactive decay of ${ }^{125}$ I on $\mathrm{Au}(111)$. Te MNN and I MNN Auger electron emission peaks are marked.

In order to further our understanding of the lack of charging, i.e. fast neutralization of the Te atom undergoing the electron emission cascades by Au we used DFT to examine the nature of the $\mathrm{Te} / \mathrm{Au}$ interaction in the $\mathrm{I} / \mathrm{Te} / \mathrm{Au}(111)$ overlayer. To this end we examined how the valence electron density rearranges upon formation of the Te/Au bond. This is shown in Figure 3 where we plot how the electron density rearranges upon subtracting from the total density of the complete $\mathrm{I} / \mathrm{Te} / \mathrm{Au}(111)$ overlayer the density of the $\mathrm{I} / \mathrm{Au}(111)$ overlayer and an isolated Te atom. This plot therefore captures how the valence electron density rearranges upon formation of the Te-Au adsorption bond in the presence of the I/Au(111) overlayer. From Figure 3 we can identify quite significant charge rearrangement around the Te and three Au atoms to which it is directly bonded. There is 
also smaller but not insignificant rearrangement of electron density in some of the second layer $\mathrm{Au}$ atoms. The charge rearrangement implies that mixed $\mathrm{Te}(\mathrm{p})-\mathrm{Au}(\mathrm{d})$ states have formed and that there is a degree of coupling between Te and the Au substrate. Indeed we have directly confirmed the formation of mixed $\mathrm{Te}(\mathrm{p})-\mathrm{Au}(\mathrm{d})$ states by analyzing the individual Kohn-Sham states in the system. We show this data in the SI in Figure SI:2. This orbital mixing and fairly long range charge rearrangement means that during the nuclear decay induced electron emission cascade the positive charge created at the $\mathrm{Te}$ atom will be spread out over many Au atoms and neutralized by the electron sea of the extended Au surface to such an extent that the effective charge on the Te atom is zero, as measured experimentally.

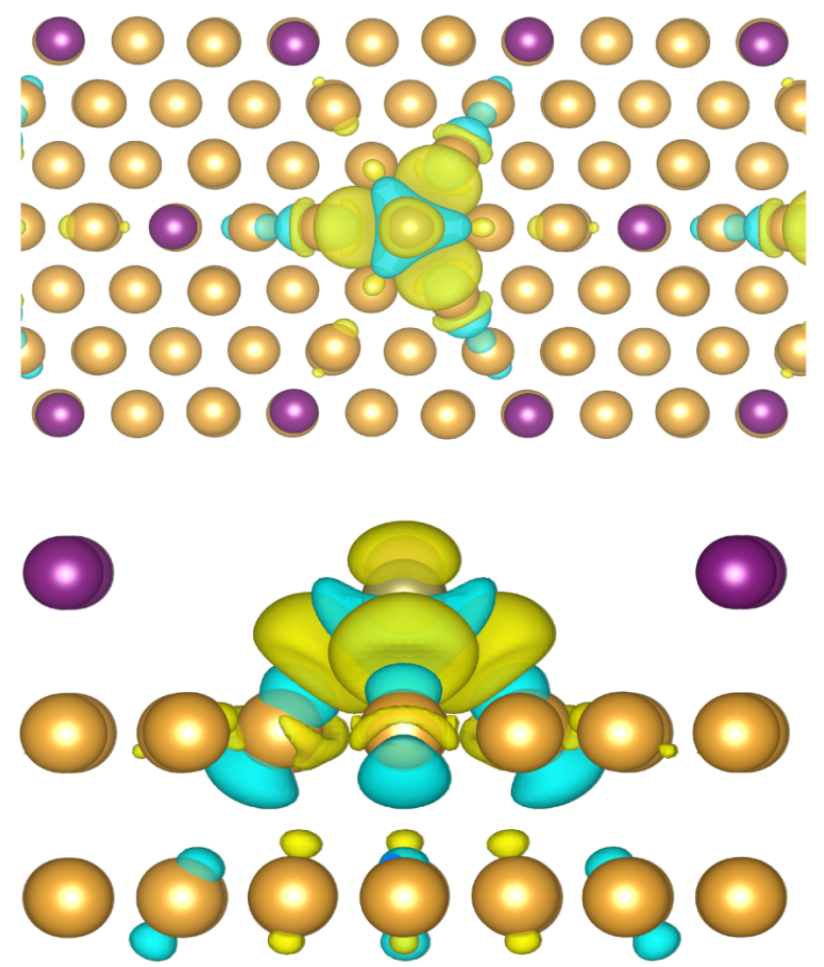

Figure 3 Electronic density rearrangement upon Te adsorption on $\mathrm{Au}(111)$. Specifically $\Delta \rho=\rho_{\text {total }}-\rho \mathrm{I} / \mathrm{Au}-\rho_{\text {Te }}$ is plotted, where $\rho_{\text {total }}$ is the charge density of the complete $\mathrm{I} / \mathrm{Te} / \mathrm{Au}(111)$ structure, $\rho_{\mathrm{I} / \mathrm{Au}}$ the charge density of the I/Au(111) overlayer and $\rho_{\text {Te }}$ the charge density of an isolated Te atom. The Te atom is located in a hollow site and interacts strongly with its three closest Au neighbors. Regions of charge increase are shown in blue, areas with charge depletion are shown in yellow, both at an isovalue of 
$0.002 \mathrm{e} / \AA^{3}$. The charge density difference from a top view and side view are shown in the upper and lower panels, respectively. For clarity, only the top two layers of the gold slab are shown. Gold atoms are colored in gold, iodine atoms in purple. The Te atom is located at the center of the charge density difference plot.

By working in UHV we are able to study the as-formed oxidation state of the ${ }^{125} \mathrm{Te}$ atom before, and then after, exposure to ambient conditions. After a few weeks of ${ }^{125} \mathrm{I}$ radioactive decay in UHV, repeated XPS measurements reveal that that the daughter Te $3 \mathrm{~d}$ core level doublet that grows over time is at $573 \mathrm{eV}$, corresponding to neutral $\mathrm{Te}^{0}$ as shown in Figure 4. ${ }^{48,49}$ All charge density decomposition schemes involve a certain level of arbitrariness, however, performing a Bader population analysis of our DFT adsorption system yields a partial charge on the Te bound to Au of $+0.22 e$, which is in good agreement with our XPS measurement and is consistent with the similar electronegativities of Au and Te. Importantly, from our DFT calculations we see that the newly formed ${ }^{125} \mathrm{Te}$ is even more tightly bound to a bare $\mathrm{Au}(111)$ surface $(3.41 \mathrm{eV})$ than ${ }^{125} \mathrm{I}(2.21 \mathrm{eV})$ which explains the film's stability during radioactive decay as the nuclear recoil energy is $\sim 0.1 \mathrm{eV}$. If the recoil energy was greater than the Te-Au bond strength the daughter Te atom could leave the surface.

The ${ }^{125} \mathrm{Te}$ atoms in the film (Te being one group to the left of I in the periodic table) are under-coordinated and, like the surface of bulk Te, are more susceptible to chemical reactions. Upon arrival from PerkinElmer the ${ }^{125} \mathrm{I}$ samples always display a small Te 3d doublet at $576 \mathrm{eV}$ and $586 \mathrm{eV},{ }^{48,49}$ which corresponds to $\mathrm{Te}^{4+}$ as shown in Figure 4 which is formed in transit. Through incremental exposure to ambient conditions and measurement by XPS we see that the newly formed $\mathrm{Te}^{0}$ undergoes oxidation to the $\mathrm{Te}^{4+}$ oxidation state. This reaction is kinetically limited as it takes about $45 \mathrm{hr}$ to fully oxidize the surface Te. To understand the details of this oxidation process we considered a range of reactions between (newly formed) Te and common species present under ambient conditions such as $\mathrm{O}_{2}$, CO, $\mathrm{H}_{2} \mathrm{O}$ and $\mathrm{H}_{2}$ with DFT (see SI for full details). Formation energies of all the species which could potentially form from reaction with atmospheric compounds are summarized in table SI:1. The most stable reaction product for each 
reaction is shown in figure SI:1. Our DFT calculations indicate that the formation of $\mathrm{TeO}_{2}$ starting from a Te atom adsorbed on Au and an oxygen molecule is exothermic by -1.24 $\mathrm{eV}$. None of the other reactions studied with DFT were as thermodynamically favorable as oxidation by molecular $\mathrm{O}_{2}$. Based on the XPS and DFT results we therefore assign the oxidized species to be $\mathrm{TeO}_{2}$.

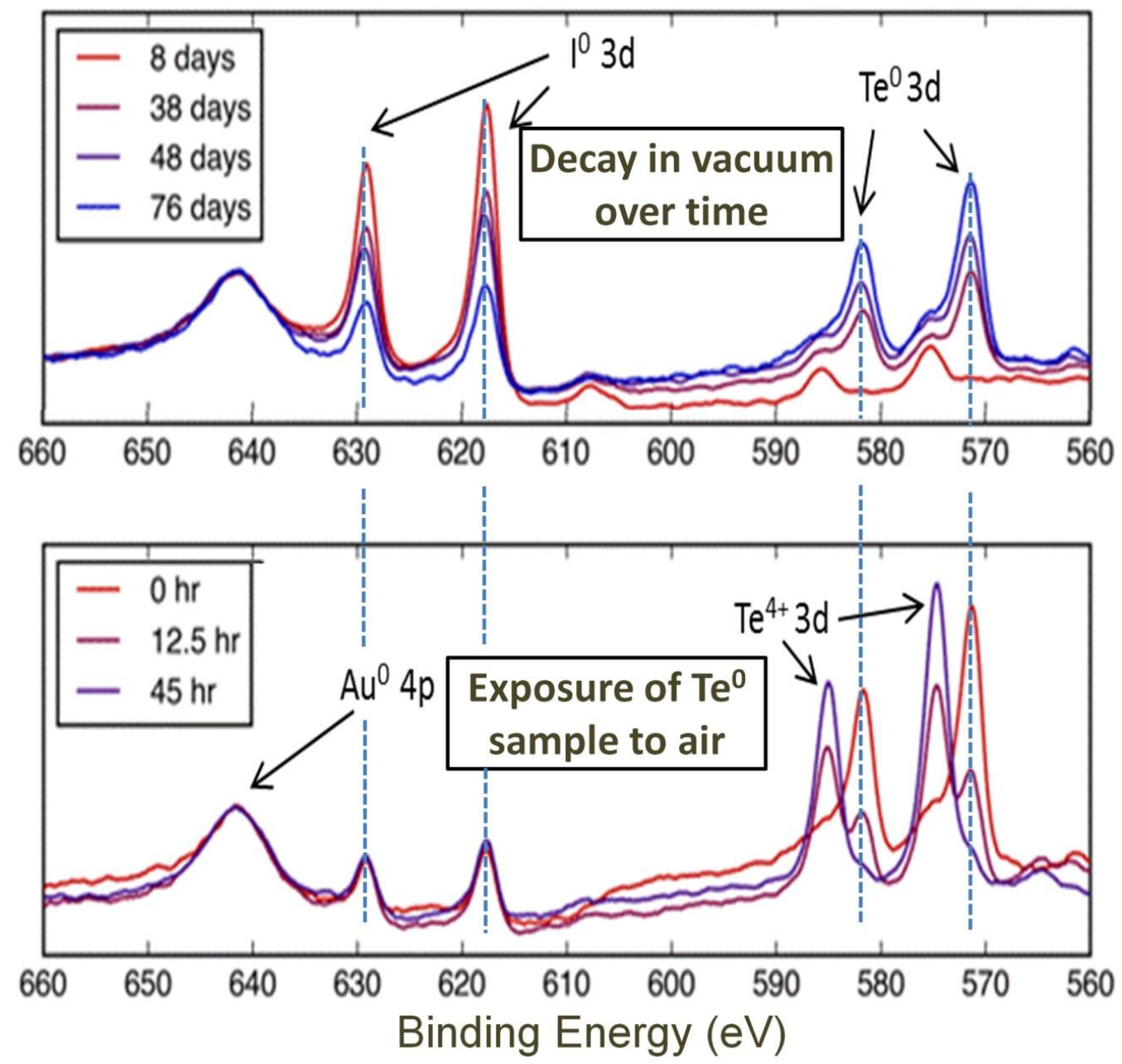

Figure 4 XPS spectra showing nuclear transmutation of I to Te and the effect of exposure to air. The upper plot shows the I $3 \mathrm{~d}$ core level signals decreasing and the $\mathrm{Te}^{0+}$ core levels increasing over time as the ${ }^{125} \mathrm{I}$ decays into ${ }^{125} \mathrm{Te}$ by electron capture. The bottom plot shows the formation of $\mathrm{Te}^{4+}$ caused by exposing a surface of $\mathrm{Te}^{0}$ formed in vacuum to ambient conditions for a number of hours.

Further support of the ambient oxidation product of Te being $\mathrm{TeO}_{2}$ comes from DFT simulated STM images. In Figure 5 we compare the DFT simulated STM images of a Te 
atom, a single $\mathrm{TeO}_{2}$ species and a $\mathrm{Te}_{2} \mathrm{O}_{4}$ dimer in a I monolayer on $\mathrm{Au}(111)$. Only the simulated image for $\mathrm{TeO}_{2}$ dimers matches the STM experiment (shown in Figure 6); we therefore assign each of the protrusions seen in the STM images as $\mathrm{TeO}_{2}$ species. The DFT computed energy difference between non-interacting $\mathrm{TeO}_{2}$ units on $\mathrm{Au}(111)$ and $\mathrm{TeO}_{2}$ dimers on $\mathrm{Au}(111)$ is $-0.1 \mathrm{eV}$, which explains why nearly all of the $\mathrm{TeO}_{2}$ imaged with STM in the form of dimers. Figure 5 (c) shows that the two $\mathrm{TeO}_{2}$ molecules are held together by a weak intermolecular Te-O bond. At $2.45 \AA$ this Te-O bond is significantly longer that the intramolecular bond which is just $1.90 \AA$. 

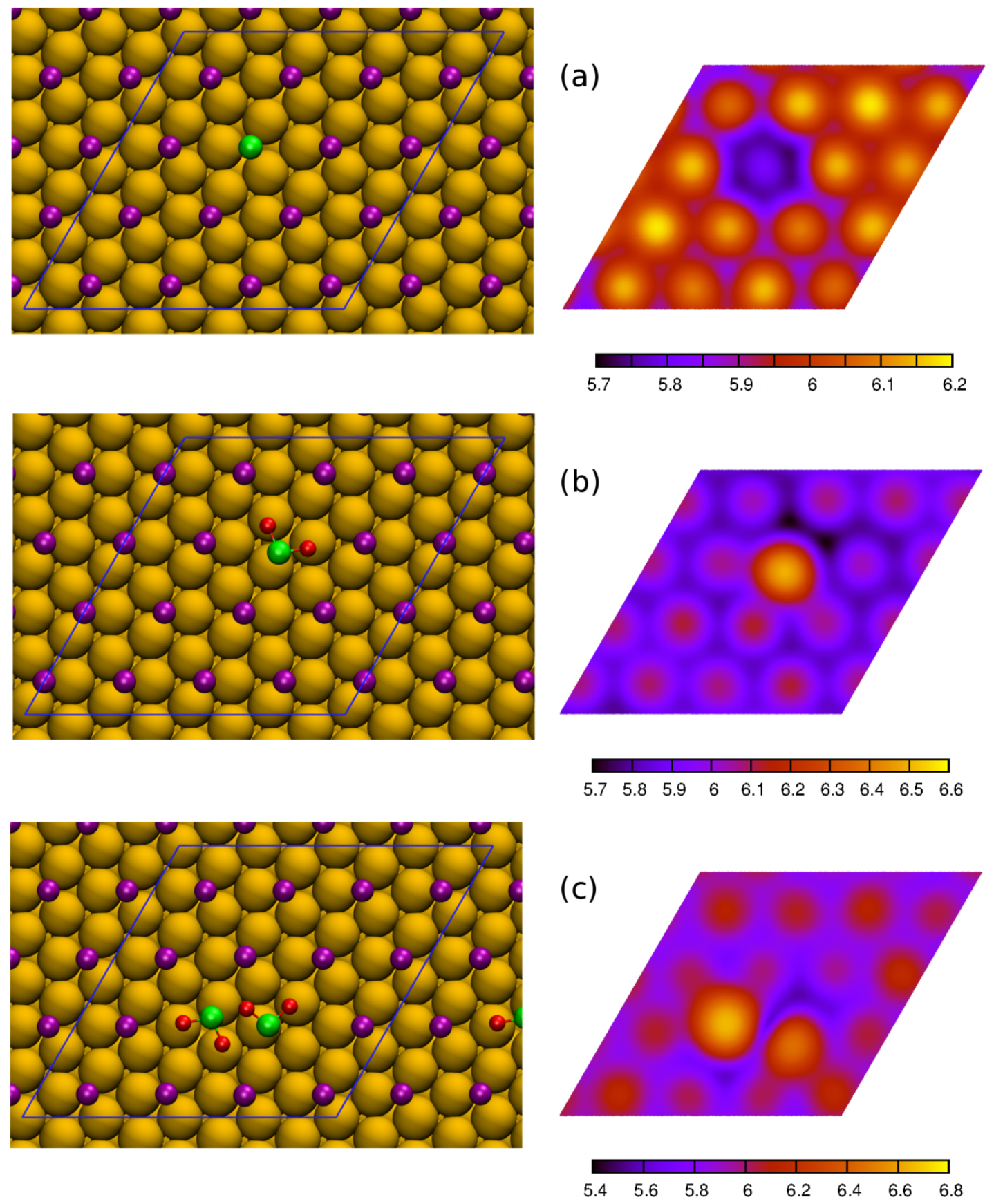

Figure 5: (a) DFT optimized structure of a Te atom (green) in an I (violet) overlayer with its corresponding simulated STM image. The Te atom appears as a depression relative to the surrounding I atoms. (b) Single $\mathrm{TeO}_{2}$ species in an I overlayer which appears as a protrusion compared to the I atoms. (c) Thermodynamically preferred $\mathrm{Te}_{2} \mathrm{O}_{4}$ and corresponding simulated STM. The dimer appears as two bright spots relative to the I atoms. Simulated STM images are shown for a constant LDOS of $1 \mathrm{e}-09 \mathrm{e} / \AA^{3}$. 
During time-lapse STM imaging of the ${ }^{125} \mathrm{I} / \mathrm{Au}(111)$ samples we observed tip-induced diffusion of the I overlayer as seen in Figure 6 . The experiments were performed at $5 \mathrm{~K}$, therefore we can rule out any effect of thermal motion. The $\mathrm{TeO}_{2}$ dimers served as "markers" in the I film that allowed us to assess the degree of mobility which is not possible in pure I overlayers on Au as there are no reference points in the overlayer. Figure 6 shows a sequence of these time-lapse images in which a $\mathrm{TeO}_{2}$ dimer moves with the I overlayer. We never observed the separation of the $\mathrm{TeO}_{2}$ dimers during these experiments, which supports their attractive interaction as predicted by DFT.

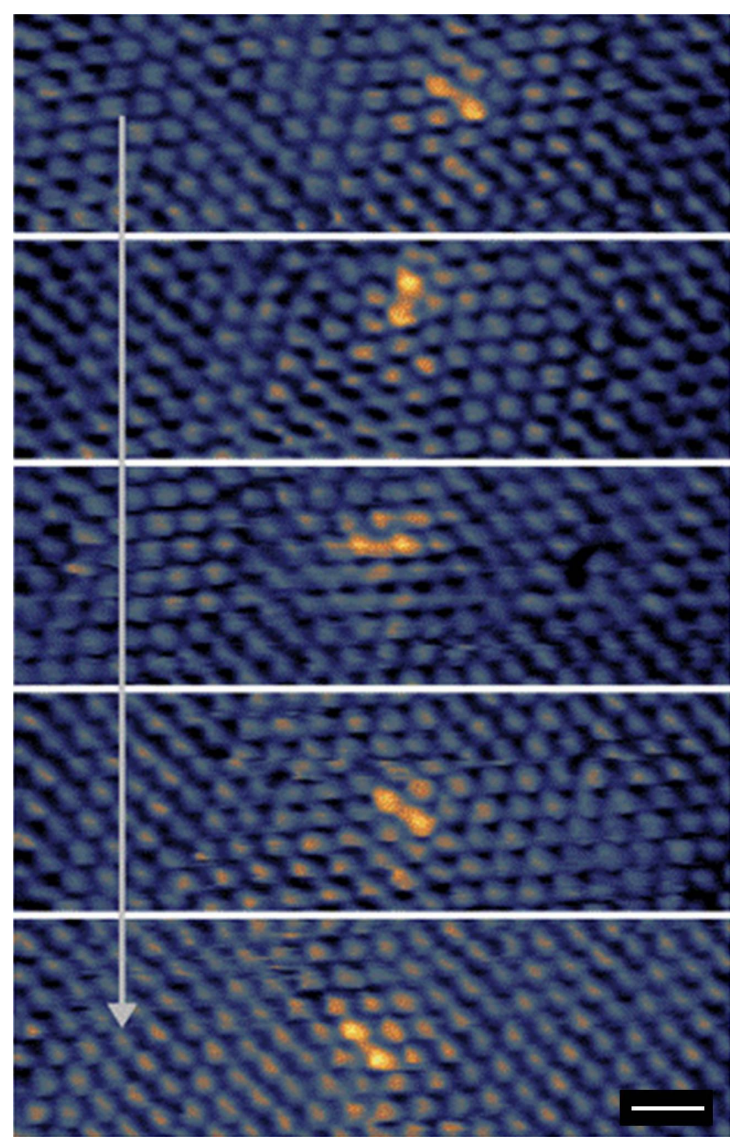

Figure 6 Diffusion of $\mathrm{TeO}_{2}$ dimer through the I monolayer. Shown is a series of consecutive STM images taken over a set location; $(\mathrm{V}=0.1 \mathrm{~V}, \mathrm{I}=0.1 \mathrm{nA}$, and scale bar is $1 \mathrm{~nm})$.

In summary, we find that ${ }^{125} \mathrm{I}$ monolayer films on $\mathrm{Au}(111)$ are remarkably stable when considering the ultra-fast release of $185 \mathrm{keV}$ of nuclear energy. This is due to the small 
nuclear recoil energy and strong hybridization with the metallic surface that prevents charging of the daughter Te atom during its electron emission cascades. DFT calculations reveal that the daughter Te atom is bound to the Au surface more strongly than the I, which itself forms a stable monolayer that is unreactive under ambient conditions. While Te is formed in its zero oxidation state XPS reveals that exposure to ambient conditions causes oxidation to the 4+ oxidation state. Extensive DFT calculations indicate that reaction with molecular oxygen is exothermic and leads to the formation of $\mathrm{TeO}_{2}$, consistent with the 4+ oxidation state measured by XPS. STM imaging and DFT simulated images indicate that the surface-bound $\mathrm{TeO}_{2}$ units pair up as $\mathrm{Te}_{2} \mathrm{O}_{4}$ dimers which, under the influence of the STM tip, freely diffuse with the I overlayer. DFT calculations show that dimer formation is exothermic as evidenced by their predominance in the STM images. These data indicate that radioactive ${ }^{125}$ I films are stable after exposure to ambient conditions and that the ${ }^{125} \mathrm{Te}$ daughter atoms are oxidized, but remain surface bound when exposed to ambient conditions.

\section{CONCLUSIONS}

The robust nature of the ${ }^{125} \mathrm{I}$ films during nuclear transmutation of I to Te bodes well for the application of radioactive films on both planar and Au nanoparticle substrates. The fact that the radioactive decay occurs via electron capture with low recoil energy renders these films robust with respect to autoradiolysis that plagues many $\alpha$ and $\beta$ emitter constructs. Despite the importance of radioactive decay in a wide range of technologies there is currently no other known air-stable 2D radiation sources. The reduced dimensionality of these surface-bound films provides the ability to study high energy nuclear processes safely and enables the microscopic details of radiation chemistry, biological degradation and material damage to be quantified. The enhanced electron emission results offer the intriguing prospect of using metal nanoparticle supported radioisotopes for the enhanced, targeted electron dosing of tumor cells with short range, chemically active low energy electrons. We envisage a new field that combines nanoscience with radioisotopes enabling understanding of many aspects of radiochemistry, physics and biology, as well as offering new constructs for in vivo radioisotope delivery for cancer therapy. Furthermore, a microscopic picture of how 
radioactive atoms can be assembled on surfaces/nanoparticles, how they decay, and how the resulting radiation affects their local molecular environment will provide fundamental knowledge about both materials and biological damage, uncover new non-equilibrium chemistries, fuel the discovery of methods for constructing nanoscale radioactive materials, and enable new technologies. 


\section{MATERIALS AND METHODS Experimental}

${ }^{125} \mathrm{I} / \mathrm{Au}(111)$ samples were made using the established ambient drop-casting method at PerkinElmer (Billerica, MA); drop-casting procedure and the resulting monolayer are shown in Figure $1 .{ }^{1}$ A solution of $\mathrm{Na}^{125} \mathrm{I}(161 \mu \mathrm{M})$ and $\mathrm{NaOH}$ in $\mathrm{H}_{2} \mathrm{O}(\mathrm{pH}$ 9) is dropped onto a clean $\mathrm{Au}(111)$ surface and dried under a constant stream of $\mathrm{N}_{2}$ gas. The ${ }^{125} \mathrm{I}$ spontaneously chemisorbs to the $\mathrm{Au}(111)$ surface, saturating when a well-ordered single layer of ${ }^{125} \mathrm{I}$ is formed. After the droplet containing ${ }^{125} \mathrm{I}$ has dried, the sample is rinsed with methanol to remove any physisorbed material. While the deposition solution has a radionuclide purity of $>99.9 \%$, it typically has an age at the time of deposition that can result in up to $30 \%$ of the ${ }^{125}$ I having already decayed into ${ }^{125} \mathrm{Te}$ i.e. a lower chemical purity. However, despite the high concentration of Te in the deposition solution, we find that the amount of Te on our ${ }^{125} \mathrm{I} / \mathrm{Au}$ samples when they first arrive at Tufts University (a few days after deposition) is no more than one would expect from the decay of a perfect ${ }^{125}$ I monolayer. This is because the newly formed Te atoms in the deposition solution are formed in a highly charged state that makes them react with water in the surrounding solution to form telluric acid/hydrated oxide which does not bind to Au. We imaged the ${ }^{125} \mathrm{I} / \mathrm{Au}(111)$ film structure with an OmicronNanotechnology low temperature STM operating at $5 \mathrm{~K}$. XPS as a function of time were taken at Tufts using a 5-channel hemispherical energy analyzer. $\mathrm{A}^{125} \mathrm{I} / \mathrm{Au}(111)$ sample was also sent to Carnegie Mellon University and used to measure high-resolution electron emission spectra using a hemispherical energy analyzer in a ThermoFisher ThetaProbe instrument. Given the importance of knowing the exact kinetic energy of the emitted electrons, and hence the oxidation state of the decaying atom, a total of 1500 individual scans were performed across the kinetic energy range from 430-530 eV with $190 \mathrm{eV}$ pass energy (constant analyzer energy). Each scan was taken using $0.2 \mathrm{eV}$ steps with a $100 \mathrm{~ms}$ dwell time per step. The kinetic energy calibration of the detector was verified by measuring the kinetic energy of electrons produced from a clean Ag standard by excitation with

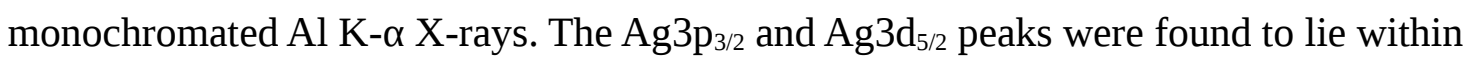
$\pm 0.1 \mathrm{eV}$ of the expected positions for metallic Ag of 913.7 and $1118.4 \mathrm{eV}$, respectively (corresponding to binding energies of 572.9 and $368.2 \mathrm{eV}$, respectively). 


\section{Theoretical}

Density functional theory (DFT) calculations were performed with the Perdew-BurkeErnzerhof (PBE) ${ }^{39,40}$ exchange-correlation functional. The periodic plane wave DFT code VASP ${ }^{41,42}$ was used to carry out all spin polarized first principle calculations. Core electrons were replaced by projector augmented wave (PAW) potentials ${ }^{43}$ and valence electrons were expanded in plane waves with a cut-off energy of $400 \mathrm{eV}$. A 4 atomic layer thick slab was used to model an unreconstructed $\mathrm{Au}(111)$ surface. The bottom two layers were kept fixed at their bulk truncated values, every other atom was allowed to relax. Slabs were separated along the surface normal with a $15 \AA$ thick vacuum region and a dipole correction along the surface normal was applied for all calculations. Total energies were converged to a $1.0 \times 10^{-6} \mathrm{eV}$ accuracy, ions were relaxed until the forces acting on them were below $0.005 \mathrm{eV} / \AA$. For all formation energy calculations a $2 \sqrt{ } 3 \times 2 \sqrt{ } 3$ unit cell was used and to explore the tendency of $\mathrm{TeO}_{2}$ molecules to dimerize a $4 \sqrt{ } 3 \times 4 \sqrt{ } 3$ cell was used. In each case a Monkhorst-Pack k-point mesh ${ }^{44}$ equivalent to at least a $6 \times 6 \times 1$ mesh per $\sqrt{ } 3 \times \sqrt{ } 3$ unit cell was used. STM images were calculated with a higher level of $\mathrm{k}$ point convergence, specifically a $24 \times 24 \times 1$ Monkhorst-Pack k-point mesh per $\sqrt{ } 3 \times \sqrt{ } 3$ unit cell at a $-0.1 \mathrm{eV}$ (i.e. looking at occupied states) bias using the Tersoff-Hamann approximation ${ }^{45}$ with an s-like tip. Additional information on the calculations performed is given in the supporting information.

\section{ACKNOWLEDGMENTS}

We are grateful to George Pappas, Garth Brown and Robert Pellegrino of PerkinElmer for providing ${ }^{125} \mathrm{I}$ and for assistance making the ${ }^{125} \mathrm{I} / \mathrm{Au}$ samples. The work at Tufts was supported the by the National Science Foundation under grant CHE-1412402 (C. M.) and by the American Chemical Society Petroleum Research Fund (54200-ND5). The work at CMU was supported by the Department of Energy (DE-FG02-12ER16330). Some of the research at UCL leading to these results has received funding from the European Research Council under the European Union’s Seventh Framework Programme (FP/2007-2013)/ERC Grant Agreement No. 616121 (HeteroIce project) and the Royal Society through a Wolfson Research merit Award (A. M.). P. P. and A. M. are grateful to the London Centre for Nanotechnology and UCL Research Computing for computational 
resources, and the UK's national high performance computing service (from which access was obtained via the UK’s Material Chemistry Consortium, EP/L000202).

\section{SUPPORTING INFORMATION AVAILABLE}

Supporting information contains additional details about path-integral DFT and KMC simulations. This material is available free of charge via the Internet at http://pubs.acs.org.

\section{TABLE OF CONTENTS GRAPHIC}

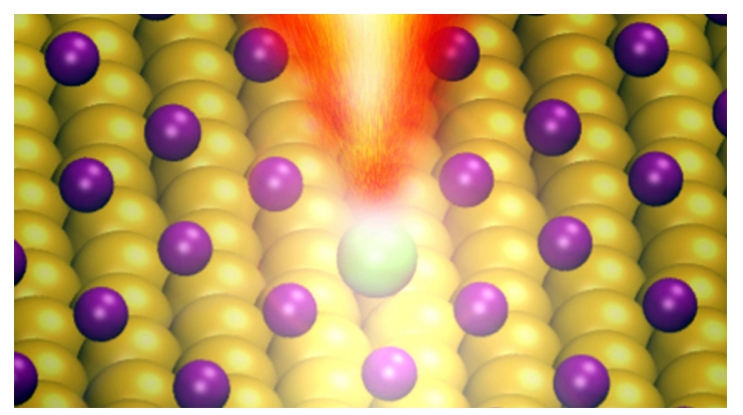

\section{References:}

(1) Pronschinske, A.; Pedevilla, P.; Murphy, C. J.; Lewis, E. A.; Lucci, F. R.; Brown, G.; Pappas, G.; Michaelides, A.; Sykes, E. C. H. Enhancement of Low-Energy Electron Emission in 2D Radioactive Films. Nat. Mater. 2015, 14, 904-907.

(2) Arumainayagam, C. R.; Lee, H.-L.; Nelson, R. B.; Haines, D. R.; Gunawardane, R. P. Low-Energy Electron-Induced Reactions in Condensed Matter. Surf. Sci. Rep. 2010, 65, 1-44.

(3) Pimblott, S. M.; LaVerne, J. A. Production of Low-Energy Electrons by Ionizing Radiation. Radiat. Phys. Chem. 2007, 76, 1244-1247.

(4) Alizadeh, E.; Sanz, A. G.; Garcia, G.; Sanche, L. Radiation Damage to DNA: The Indirect Effect of Low-Energy Electrons. J. Phys. Chem. Lett. 2013, 4, 820-825.

(5) Simons, J. How Do Low-Energy (0.1-2 eV) Electrons Cause DNA Strand Breaks? Acc. Chem. Res. 2007, 963, 256-259.

(6) Barrios, R.; Skurski, P.; Simons, J. Mechanism for Damage to DNA by LowEnergy Electrons. J. Phys. Chem. B 2002, 106, 7991-7994.

(7) Boudaïffa, B.; Cloutier, P.; Hunting, D.; Huels, M. A.; Sanche, L. Resonant Formation of DNA Strand Breaks by Low-Energy (3 to $20 \mathrm{eV}$ ) Electrons. Science 2000, 287, 1658-1660. 
(8) Alexander, W. A.; Wiens, J. P.; Minton, T. K.; Nathanson, G. M. Reactions of Solvated Electrons Initiated by Sodium Atom Ionization at the Vacuum-Liquid Interface. Science 2012, 335, 1072-1075.

(9) Alizadeh, E.; Sanche, L. Precursors of Solvated Electrons in Radiobiological Physics and Chemistry. Chem. Rev. 2012, 112, 5578-5602.

(10) Siefermann, K. R.; Liu, Y.; Lugovoy, E.; Link, O.; Faubel, M.; Buck, U.; Winter, B.; Abel, B. Binding Energies, Lifetimes and Implications of Bulk and Interface Solvated Electrons in Water. Nat. Chem. 2010, 2, 274-279.

(11) Garrett, B. C.; Dixon, D. A.; Camaioni, D. M.; Chipman, D. M.; Johnson, M. A.; Jonah, C. D.; Kimmel, G. A.; Miller, J. H.; Rescigno, T. N.; Rossky, P. J.; Xantheas, S. S.; Colson, S. D.; Laufer, A. H.; Ray, D.; Barbara, P. F.; Bartels, D. M.; Becker, K. H.; Bowen, K. H.; Bradforth, S. E.; Carmichael I.; et al. Role of Water in Electron-Initiated Processes and Radical Chemistry: Issues and Scientific Advances. Chem. Rev. 2005, 105, 355-389.

(12) Balagurumoorthy, P.; Xu, X. Effect of Distance between Decaying 125I and DNA on Auger-Electron Induced Double-Strand Break Yield. Int. J. Radiat. Biol. 2012, 88, 998-1008.

(13) Hervé Du Penhoat, M. A.; Huels, M. A.; Cloutier, P.; Jay-Gerin, J. P.; Sanche, L. Electron Stimulated Desorption of H- from Thin Films of Thymine and Uracil. $J$. Chem. Phys. 2001, 114, 5755-5764.

(14) Hainfeld, J. F.; Slatkin, D. N.; Smilowitz, H. M. The Use of Gold Nanoparticles to Enhance Radiotherapy in Mice. Phys. Med. Biol. 2004, 49, N309-N315.

(15) Zheng, Y.; Hunting, D. J.; Ayotte, P.; Sanche, L. Radiosensitization of DNA by Gold Nanoparticles Irradiated with High-Energy Electrons. Radiat. Res. 2008, 169, 19-27.

(16) Martin, F.; Burrow, P. D.; Cai, Z.; Cloutier, P.; Hunting, D.; Sanche, L. DNA Strand Breaks Induced by 0-4 eV Electrons: The Role of Shape Resonances. Phys. Rev. Lett. 2004, 93, 6-9.

(17) Kim, Y. H.; Jeon, J.; Hong, S. H.; Rhim, W. K.; Lee, Y. S.; Youn, H.; Chung, J. K.; Lee, M. C.; Lee, D. S.; Kang, K. W.; Nam, J-M. Tumor Targeting and Imaging Using Cyclic RGD-PEGylated Gold Nanoparticle Probes with Directly Conjugated Iodine-125. Small 2011, 7, 2052-2060.

(18) Dam, D. H. M.; Lee, J. H.; Sisco, P. N.; Co, D. T.; Zhang, M.; Wasielewski, M. R.; Odom, T. W. Direct Observation of Nanoparticle-Cancer Cell Nucleus Interactions. ACS Nano 2012, 6, 3318-3326.

(19) Gu, Y. J.; Cheng, J.; Lin, C. C.; Lam, Y. W.; Cheng, S. H.; Wong, W. T. Nuclear Penetration of Surface Functionalized Gold Nanoparticles. Toxicol. Appl. Pharmacol. 2009, 237, 196-204.

(20) Bardhan, R.; Lal, S.; Joshi, A.; Halas, N. J. Theranostic Nanoshells: From Probe Design to Imaging and Treatment of Cancer. Acc. Chem. Res. 2011, 44, 936-946.

(21) Ghosh, P.; Han, G.; De, M.; Kim, C. K.; Rotello, V. M. Gold Nanoparticles in Delivery Applications. Adv. Drug Deliv. Rev. 2008, 60, 1307-1315. 
(22) Craig, G. E.; Brown, S. D.; Lamprou, D. A.; Graham, D.; Wheate, N. J. CisplatinTethered Gold Nanoparticles That Exhibit Enhanced Reproducibility, Drug Loading, and Stability: A Step Closer to Pharmaceutical Approval? Inorg. Chem. 2012, 51, 3490-3497.

(23) Cheng, W.; Dong, S.; Wang, E. Iodine-Induced Gold-Nanoparticle Fusion/fragmentation/aggregation and Iodine-Linked Nanostructured Assemblies on a Glass Substrate. Angew. Chemie - Int. Ed. 2003, 42, 449-452.

(24) Huang, L.; Huang, L.; Zeppenfeld, P.; Zeppenfeld, P.; Horch, S.; Horch, S.; Comsa, G.; Comsa, G. Determination of Iodine Adlayer Structures on Au(111) by Scanning Tunneling Microscopy. J. Chem. Phys. 1997, 107, 585.

(25) Yamada, T.; Batina, N.; Itaya, K. Structure of Electrochemically Deposited Iodine Adlayer on Au(111) Studied by UltrahighVacuum Instrumentation and in Situ STM. J. Phys. Chem. 1995, 99, 8817-8823.

(26) Zhu, L. Neutrino-Recoil Induced Desorption. J. Vac. Sci. Technol. A Vacuum, Surfaces, Film. 1994, 12, 2037.

(27) McLaughlin, M. F.; Woodward, J.; Boll, R. A.; Wall, J. S.; Rondinone, A. J.; Kennel, S. J.; Mirzadeh, S.; Robertson, J. D. Gold Coated Lanthanide Phosphate Nanoparticles for Targeted Alpha Generator Radiotherapy. PLoS One 2013, 8, 2-9.

(28) Hsiung, C.; Gordus, A. A. Momentum Transfer to an Atom in a Molecule: Internal Excitation and Bond Dissociation. J. Chem. Phys. 1962, 36, 947.

(29) Booth, C. H.; Bauer, E. D.; Daniel, M.; Wilson, R. E.; Mitchell, J. N.; Morales, L. A.; Sarrao, J. L.; Allen, P. G. Quantifying Structural Damage from Self-Irradiation in a Plutonium Superconductor. Phys. Rev. B - Condens. Matter Mater. Phys. 2007, $76,1-7$.

(30) Farnan, I.; Cho, H.; Weber, W. J. Quantification of Actinide Alpha-Radiation Damage in Minerals and Ceramics. Nature 2007, 445, 190-193.

(31) Suggs, D. W.; Stickney, J. L. Characterization of Atomic Layers of Tellurium Electrodeposited on the Low-Index Planes on Gold. J. Phys. Chem. 1991, 95, 10056-10064.

(32) Sorenson, T. A. A Comparison of Atomic Layers Formed by Electrodeposition of Selenium and Tellurium Scanning Tunneling Microscopy Studies on Au(100) and Au(111). J. Electrochem. Soc. 1999, 146, 1019.

(33) Verkhoturov, S. V; Schweikert, E. a; Chechik, V.; Sabapathy, R. C.; Crooks, R. M.; Parilis, E. S. Auger Stimulated Ion Desorption of Negative Ions via K-Capture Radioactive Decay. Phys. Rev. Lett. 2001, 87, 037601.

(34) Gokhberg, K.; Kolorenč, P.; Kuleff, A. I.; Cederbaum, L. S. Site- and EnergySelective Slow-Electron Production through Intermolecular Coulombic Decay. Nature 2014, 505, 661-663.

(35) Nath, A.; Prushan, M. J.; Gilbert, J. G. Can Super-Excited Molecules Survive Fragmentation? J. Radioanal. Nucl. Chem. 2001, 247, 589-591. 
(36) Carlson, T. A.; White, R. M. Formation of Fragment Ions from CH3Te125 and C2H5Te125 Following the Nuclear Decays of CH3I125 and C2H5I125. J. Chem. Phys. 1963, 38, 2930.

(37) Pomplun, A. E.; Booz, J.; Charlton, D. E.; Pomplun, E. A Monte Carlo Simulation of Auger Cascades. Radiat. Res. 1987, 111, 533-552.

(38) Kolasinski, K. Surface Science: Foundations of Catalysis and Nanoscience; Wiley, 2012.

(39) Perdew, J. P.; Burke, Kieron; Ernzerhof, M. Generalized Gradient Approximation Made Simple. Phys. Rev. Lett. 1996, 77, 3865.

(40) Perdew, J. P.; Burke, K.; Ernzerhof, M. Generalized Gradient Approximation Made Simple- ERRATA. Phys. Rev. Lett. 1996, 77, 3865-3868.

(41) Kresse, G.; Hafner, J. Ab Initio Molecular Dynamics of Liquid Metals. Physical Review B: Condensed Matter and Materials Physics, 1993, 47, 558-561.

(42) Kresse, G.; Furthmüller, J. Efficient Iterative Schemes for Ab Initio Total-Energy Calculations Using a Plane-Wave Basis Set. Phys. Rev. B 1996, 54, 11169-11186.

(43) Kresse, G.; Joubert, D. From Ultrasoft Pseudopotentials to the Projector Augmented-Wave Method. Phys. Rev. B 1999, 59, 1758-1775.

(44) Monkhorst, H. J.; Pack, J. D. Special Points for Brillouin-Zone Integrations. Phys. Rev. B 1976, 13, 5188-5192.

(45) Tersoff, J.; Hamann, D. R. Theory of the Scanning Tunneling Microscope. Phys. Rev. B 1985, 31, 805-813.

(46) Charlton, D. E.; Booz, J. A Monte Carlo Treatment of the Decay of 125I. Radiat. Res. 1981, 87, 10-23.

(47) Bahl, M. K.; Watson, R. L.; Irgolic, K. J. X-Ray Photoemission Studies of Tellurium and Some of Its Compounds. J. Chem. Phys. 1977, 66, 5526.

(48) Detty, M.; Lenhart, W. C.; Gassman, P. G.; Callstrom, M. R. XPS and 125Te NMR Studies of Organotellurium Compounds. 1. Tellurapyrans, Tellurapyranones, Tellurapyrylium Salts, and Their Benzo Analogues in Both the Tellurium(II) and Tellurium(IV) Oxidation States. Organometallics 1989, 8, 861-865.

(49) Chastain, J.; Moulder, J. F. Handbook of X-Ray Photoelectron Spectroscopy: A Reference Book of Standard Spectra for Identification and Interpretation of XPS Data; Physical Electronics, 1995.

(50) Klimeš, J.; Bowler, D. R.; Michaelides, A. Van Der Waals Density Functionals Applied to Solids. Phys. Rev. B 2011, 83, 195131. 